\title{
Gastar Dinheiro em Roupas no Shopping: Os Significados de "Comprar" para Adolescentes Brasileiros e Portugueses
}

\author{
Samuel Lincoln Bezerra Lins ${ }^{\mathbf{1}}$ \\ Departamento de Psicologia da Pontifícia Universidade Católica do Rio de Janeiro, \\ Rio de Janeiro, RJ, Brasil \\ Gabrielle Poeschl \\ Centro de Psicologia da Universidade do Porto, Porto, Portugal
}

\begin{abstract}
Resumo
A adolescência é um período de conflitos onde o consumo desempenha um papel importante na construção da identidade pessoal e social. Contudo, apesar da vasta investigação sobre o comportamento de consumo, pouca atenção parece ter sido dada aos adolescentes. Esta investigação teve o objectivo de conhecer os significados que rapazes e raparigas atribuem ao acto de comprar, bem como o de analisar a influência que o sexo de pertença e o contexto socioeconómico têm sobre estes significados. O contexto socioeconómico considerado foi, no Brasil, o tipo de escola, em Portugal, a percepção da crise económica, sendo ambos os factores ligados a diferenças sociais. A amostra do primeiro estudo foi composta por 482 adolescentes brasileiros, sendo 272 alunos da escola privada (129 rapazes e 143 raparigas) e 210 alunos da escola pública (84 rapazes e 126 raparigas). No segundo estudo participaram 238 adolescentes portugueses de uma escola pública (117 rapazes e 121 raparigas). Os resultados indicam que existe uma grande homogeneidade nos significados atribuídos ao acto de "comprar" no público adolescente, e que o sexo de pertença, associado a diferentes tipos de produtos, modula mais do que o contexto socioeconómico esses significados.
\end{abstract}

Palavras-chave: Significados, associação livre de palavras, consumo, adolescência.

\section{Spending Money in Clothes within the Shopping Mall: The Meanings of "Buying" for Brazilian and Portuguese Adolescents}

\begin{abstract}
Adolescence is a time of conflicts in which consumption plays an important role in the construction of personal and social identity. However, in spite of the extensive research on consumption behavior, not much attention has been given to adolescents. This research aimed to uncover the meanings that boys and girls associate to the buying behavior, as well as to analyze the effect that sex belongingness and socioeconomic status have on these meanings. The effect of the socioeconomic context was studied, in Brazil, through the type of school, and, in Portugal, through the perception of the economic crisis, being both factors related to social differences. In the first study participants were 482 Brazilian adolescents,
\end{abstract}

Endereço para correspondência: Pontifícia Universidade Católica do Rio de Janeiro, Departamento de Psicologia, Gávea, Rio de Janeiro, RJ, Brasil 22451-900. Fone: (21) 3527-1001. E-mail: samuel.bezerra.lins@ gmail.come gpoeschl@fpce.up.pt

Esta pesquisa foi financiada pelo Programa Erasmus Mundus External Cooperation Window (Projecto MUNDUS17 - coordenado pela Universidade do Porto). 
272 pupils from a private school (129 boys and 143 girls) and 210 pupils from a public school ( 84 boys and 126 girls). In the second study, respondents were 238 Portuguese adolescents from a public school (117 boys and 121 girls). Results reveal that there is a high homogeneity in the meanings attributed to the act of "buying" by the adolescent public, and that sex belongingness, associated to different types of products, modulate these meanings more than the socioeconomic context.

Keywords: Meanings, word association, consumption, adolescence.

\section{Gastar Dinero en la Ropa en el Centro Comercial: Los Significados de "Compra" para los Adolescentes Brasileños y Portugueses}

\section{Resumen}

La adolescencia es un período de conflictos, donde el consumo desempeña un papel importante en la construcción de la identidad personal y social. Sin embargo, a pesar de una amplia investigación sobre el comportamiento del consumidor, parece poca la atención que se ha dado a los adolescentes. Esta investigación tuvo como objetivo comprender los significados que los niños y niñas atribuyen al acto de compra, así como analizar la influencia que el sexo de pertenencia y el contexto socio-económico tienen sobre estos significados. El contexto socio-económico considerado fue, en Brasil, el tipo de escuela, y en Portugal, la percepción de la crisis económica, siendo ambos factores ligados a las diferencias sociales. La muestra del primero estudio estuvo compuesta por 482 adolescentes brasileños, siendo 272 estudiantes de la escuela privada (129 niños y 143 niñas) y 210 estudiantes de la escuela pública (84 niños y 126 niñas). En el segundo estudio, 238 adolescentes portugueses de una escuela pública (117 niños y 121 niñas). Los resultados indican que existe una gran uniformidad en los significados atribuidos a "comprar" en el público adolescente, y que el sexo de pertenencia, relacionado con los distintos tipos de productos, modula más que el contexto socio-económico de estos significados.

Palabras clave: Significados, asociación libre de palabras, consumo, adolescencia.

Encontramos referências sobre discussões relativas aos comportamentos económicos desde as civilizações gregas e romanas. No que diz respeito, mais particularmente, ao termo "consumo", este aparece já em 1910 na $11^{\text {a }}$ edição da Enciclopédia Britânica onde é definido como desperdício, no sentido físico, e como falência de empresas públicas, no vocabulário técnico da economia (Bauman, 2007). O consumo, na sua acepção actual em microeconomia designa o acto que permite concretizar a satisfação de determinada necessidade através da utilização de determinado bem (Garófalo, 1998), e é precisamente o comportamento ligado à aquisição de bens e serviços que, nos dias de hoje, está no centro dos estudos do consumo. Este campo de investigação tem-se desenvolvido em diversas áreas de conhecimento, como a economia, a sociologia, a antropologia, a comunicação social, e a psicologia (Amon, Guareschi, Roso, \& Vero- nese, 2010; Hands, 2010; Lillis, 2008; Macinnis $\&$ Folkes, 2010), tornando-se um campo de estudo interdisciplinar.

Os estudos realizados constataram que o consumo desempenha actualmente um papel central na vida dos indivíduos, principalmente no público adolescente que tende a valorizar mais as posses e os bens materiais do que outro grupo etário e é mais vulnerável à influência da publicidade que satura os meios de comunicação social (Santos \& Fernandes, 2011). Para compreender melhor o comportamento de compra dos adolescentes, procurámos conhecer os significados que os adolescentes brasileiros e portugueses atribuem ao acto de comprar.

\section{Os Adolescentes e o Consumo}

Os jovens consumidores de hoje não são apenas criados numa sociedade do consumo 
mas eles têm também mais dinheiro disponível do que qualquer outro grupo de adolescentes na história (Castro, 1998; Morton, 2002; Noble, Haytko, \& Philips, 2009). O aumento do poder de compra dos adolescentes explica assim o interesse do mercado em atrair este público para consumir os seus produtos e serviços (Gunter, 2001). Esta mudança explica que, se dantes ter adolescentes perto das lojas representava um inconveniente por ter um impacto negativo na segurança da loja, actualmente isto já não é o caso (Mangleburg, Doney, \& Bristol, 2004). Sabe-se também que as experiências de consumo vivenciadas na adolescência são importantes para a construção dos padrões de comportamentos dos jovens consumidores, e o desenvolvimento de hábitos de consumo que serão adoptados na fase adulta (Ward, 1974).

Os adolescentes têm ainda maior probabilidade de aderir e de acompanhar as tendências do mercado (Latif, Saleem, \& Abideen, 2011). Além de influenciarem os seus pares, os adolescentes são formadores de opinião da população em geral (Latif et al., 2011; Noble et al., 2009). Eles influenciam as despesas dos seus pais, participando de forma significativa nas decisões de compra de toda a família (Latif et al., 2011; Mangleburg et al., 2004; Wang, Holloway, Beatty, \& Hill, 2007). Acrescenta-se que cada vez mais são atribuídas tarefas de compra aos adolescentes no ambiente familiar porque, além de terem mais tempo livre, eles também gostam mais de fazer compras do que seus pais (Bush, Martin, \& Bush, 2004).

Apesar da sua relevância, o público consumidor adolescente, as suas compras, as suas relações com os produtos, e o modo como percebe o valor das marcas, parecem ser áreas ainda relativamente pouco exploradas (Chandler \& Heinzerling, 1999).

\section{Rapazes e Raparigas}

As expectativas sociais sobre o desempenho do papel masculino e feminino já exercem precocemente uma função importante na construção da subjectividade de homens e mulheres. O sexo de pertença do adolescente influencia a forma como ele lida com as suas emoções, a relação com o grupo de amigos, os conflitos e relacionamentos familiares, e a sua perspectiva de futuro (Ozella \& Aguiar, 2008). Além disso, o sexo de pertença do adolescente também afecta o seu comportamento de consumo (Moschis, Moore, \& Stephens, 1977; Shannahan, Shannahan, Bush, \& Rocco, 2011).

É importante sublinhar que as diferenças de sexo não devem ser percebidas com uma visão essencialista. A relação entre sexo e consumo é o resultado da internalização de normas socioculturais e representações socialmente compartilhadas (Dittmar, 2011). Desde cedo as crianças internalizam as representações de género estabelecidas pela sociedade vinculando determinadas categorias de objectos ao sexo de pertença (Crabb \& Bielawski, 1994). Como resultado desta internalização, rapazes e raparigas compram categorias de produtos diferentes (Dittmar, 1989) e aprendem a usar e a possuir produtos por motivações sociais diferentes (Lloyd \& Duveen, 1990).

\section{Contextos Socioeconómicos}

Como é óbvio, as variáveis financeiras exercem uma influência nas compras dos jovens (Cassidy \& Schijndel, 2011; Hall, 2002), ou seja, o estatuto socioeconómico dos adolescentes vai influenciar os seus padrões de comportamento de compra. Além disso, as motivações de compra dos adolescentes e a decisão sobre os produtos que desejam adquirir são influenciadas pelas normas da classe social na qual estão inseridos (Gade, 1998; Shim, Serido, \& Barber, 2011).

Partindo destas constatações, a nossa investigação teve o objectivo de conhecer os significados que rapazes e raparigas atribuem ao acto de comprar e de identificar eventuais factores de variação nestes significados que surgem dos contextos socioeconómicos que os adolescentes vivenciam no seu país.

Observa-se que, Brasil e Portugal, apesar de ser dois países com grandes semelhanças históricas e culturais, vivenciam actualmente situações económicas completamente diferentes. Enquanto o Brasil está a experimentar um desenvolvi- 
mento económico como nunca antes visto na sua história, Portugal tem vivido um período de intensa crise económica com elevados índices de desemprego. Procurámos, portanto, examinar em que medida o acto de comprar tem significados semelhantes ou diferentes para a generalidade dos adolescentes, inseridos em contextos socioeconómicos diferentes.

O contexto nacional considerado no Brasil foi o tipo de escola (pública ou privada) em que os jovens estudam. Com efeito, comparar escolas públicas e privadas no Brasil, significa comparar alunos de realidades económicas e sociais distintas, pois:

cada tipo de instituição escolar atende alunos de bases socioeconómicas diferentes . . . o tipo de escola e as desigualdades na condição de acesso ao ensino de qualidade já indicam a diferenciação do nível socioeconómico das famílias dos alunos da rede de ensino público. (Camargo \& Bertoldo, 2006, p. 371)

Outras investigações também apontam neste sentido (Costa \& Koslinski, 2012; França \& Gonçalves, 2010; Pasdiora \& Brei, 2014; Sampaio \& Guimarães, 2009).

O contexto nacional considerado em Portugal foi a percepção da crise económica. Com efeito, diferentes níveis de percepção da crise económica e a preocupação com as suas consequências influenciam directamente o comportamento de compra das pessoas, e os seus hábitos de consumo (Roland-Levy, Boumelki, \& Guillet, 2010).

\section{Método}

\section{Amostra}

No primeiro estudo participaram 482 alunos brasileiros do ensino médio de uma escola pública e de uma escola privada da cidade de João Pessoa (Paraíba, Brasil). Da escola privada, participaram 272 adolescentes do $9^{\circ}$ ao $11^{\circ}$ ano, 129 rapazes e 143 raparigas, com uma idade média de 14,49 anos $(D P=1,01)$. Da escola pública participaram 210 adolescentes do $10^{\circ}$ ao $12^{\circ}$ ano, 84 rapazes e 126 raparigas, com uma idade média de 15,43 anos $(D P=1,13)$.
No segundo estudo participaram 238 adolescentes portugueses, 117 rapazes e 121 raparigas com uma idade média de 15,43 anos ( $D P$ $=1,76)$, também alunos do ensino médio, do $8^{\circ}$ ao $12^{\circ}$ ano, de uma escola pública localizada na cidade de Maia (distrito de Porto, Portugal).

\section{Instrumentos}

O estudo foi conduzido por meio de um questionário utilizando a técnica de associação livre de palavras. Assim, na primeira página, pedia-se aos alunos para responder à seguinte questão:

Quando você pensa na palavra "COMPRAR”, quais são as 5 primeiras palavras ou expressões que the vêm espontaneamente à mente? Depois, para cada resposta que você escreveu, você deve dizer se ela tem um sentido negativo ou positivo, colocando o número de 1 a 5 , que melhor represente a sua resposta.

Para avaliar a percepção da crise económi$c a$ dos adolescentes portugueses, solicitámos aos participantes que indicassem o quanto ( 1 = nada; 7 = extremamente) sentiam que a actual crise económica afectava directamente (1) a sua vida; (2) a vida da sua família; (3) a vida dos seus amigos; e (4) Portugal. De seguida recolhia-se alguns dados sociodemográficos (idade, sexo, e ano escolar).

\section{Procedimentos}

Os alunos foram informados sobre os objectivos do estudo, como também sobre o anonimato e sigilo das informações, garantido o carácter voluntário da participação. Depois de se obter os termos de consentimento, os inquéritos foram aplicados em ambiente de sala de aula. No Brasil os inquéritos foram aplicados nos meses de Fevereiro e Maio de 2011, e em Portugal, no mês de Maio de 2012. O projecto de pesquisa foi aprovado pela Comissão de Ética da Faculdade de Psicologia e Ciências das Educação da Universidade do Porto (Portugal).

\section{Análise dos Dados}

Os dados foram tratados de acordo com as regras clássicas de redução das respostas às ta- 
refas de associação livre (Rosenberg \& Jones, 1972), como reduzir as palavras à forma masculina singular, transformar os verbos para a sua forma infinitiva, eliminar as palavras "ferramenta" (por exemplo: artigos e proposições), excluir os moderadores, trocar os advérbios e substantivos pelos adjectivos correspondentes. Adicionalmente, as palavras de significado semelhante foram reunidas à sua forma mais comum, com a concordância de três juízes independentes.

As palavras recolhidas por meio do questionário foram transcritas e calculou-se a frequência de cada palavra citada. Para examinar os efeitos do sexo, da escola de pertença (no Brasil) e da percepção da crise (em Portugal), aplicou-se o teste de qui-quadrado sobre as frequências das palavras. Adicionalmente, realizou-se uma análise de variância (ANOVA $2 \times 2$ ) sobre a sua positividade, a fim de verificar se havia alguma interacção entre o sexo e o contexto socioeconómico.

Também calculou-se o índice de riqueza (razão entre o número de palavras diferentes evocadas e o número total de palavras recolhidas), que mede a integração da informação acerca de um objecto. $\mathrm{O}$ índice de riqueza pode variar de 0 a 1 , ou seja, do consenso total à divergência total (Deconchy, 1971; Poeschl, 1992). Por fim, foi calculada a positividade das palavras, representada pela média da positividade de todas as palavras evocadas pelos participantes.

\section{Resultados}

\section{Estudo 1: Brasil}

No total foram recolhidas 2337 respostas à questão de saber o que os adolescentes pensam quando pensam em "comprar", entre as quais 329 foram palavras diferentes. O índice de riqueza é baixo $(0,14)$, o que revela uma representação estereotipada do conceito de "comprar". As frequências de evocação variam entre 1 (161 palavras únicas) e 283 ("roupa"). Apresentamos de seguida os resultados relativos às palavras mais citadas, e comparamos os resultados em função do sexo (masculino vs. feminino) e do tipo de escola (privada $v s$. pública).

\section{Resultado Global}

As dez palavras mais citadas pelos adolescentes brasileiros resultantes das associações livres foram: roupa $(n=283)$, dinheiro $(n=222)$, sapato $(n=127)$, shopping $(n=122)$, gastar $(n$ $=74)$, loja $(n=72)$, comida $(n=59)$, bolsa $(n=$ $51)$, carro $(n=50)$ e diversão $(n=33)$.

\section{Resultado por Sexo}

As cinco palavras mais citadas pelos rapazes brasileiros foram: roupa $(n=101)$, dinheiro ( $n=98)$, shopping $(n=48)$, gastar $(n=38)$ e loja $(n=33)$. As cinco palavras mais citadas pelas raparigas brasileiras foram: roupa $(n=182)$, dinheiro $(n=124)$, sapato $(n=102)$, shopping ( $n$ $=74)$ e bolsa $(n=48)$. A fim de verificar se havia diferenças significativas no que diz respeito à frequência das palavras evocadas pelos alunos do sexo masculino e do sexo feminino, aplicou-se um teste de qui-quadrado sobre as palavras que obtiveram frequência igual ou superior a 10 , totalizando 34 palavras (ver Tabela 1).

Das 34 palavras mais evocadas, apenas 12 apresentaram diferenças significativas nas frequências relativas ao sexo. Os rapazes evocaram mais as palavras como: carro $(p<0,01)$, computador $(p<0,01)$, sapatilha $(p<0,001)$, videojogos $(p<0,001)$, e filme $(p<0,05)$, enquanto as raparigas evocaram mais as palavras como: roupa $(p<0,01)$, sapato $(p<0,001)$, bolsa $(p<0,001)$, acessórios $(p<0,001)$, maquilhagem $(p<0,01)$, jóias $(p<0,05)$, e perfume $(p<0,05)$.

\section{Resultado por Escola}

As cinco palavras mais citadas pelos alunos da escola privada foram: roupa $(n=148)$, dinheiro $(n=141)$, shopping $(n=100)$, loja $(n=61) \mathrm{e}$ sapato $(n=54)$. As cinco palavras mais citadas pelos alunos de escola pública foram: roupa $(n=$ $135)$, dinheiro $(n=81)$, sapato $(n=73)$, comida $(n=31)$ e carro $(n=28)$.

Com o objectivo de saber se havia diferenças significativas na frequência das palavras evocadas pelos alunos da escola privada e da escola pública, aplicou-se novamente o teste de qui-quadrado (ver Tabela 2). 
Tabela 1

Frequência das Palavras mais Evocadas $(f>10)$ pelos Respondentes Brasileiros em Função do Sexo de Pertença

\begin{tabular}{|c|c|c|c|c|c|}
\hline & Palavras & $\begin{array}{l}\text { Rapazes } \\
n=213\end{array}$ & $\begin{array}{c}\text { Raparigas } \\
n=269\end{array}$ & $\begin{array}{c}\text { Total } \\
n=482\end{array}$ & $\begin{array}{c}\chi^{2} \\
(g l=1)\end{array}$ \\
\hline 1 & Roupa & 101 & 182 & 283 & $8,29 * *$ \\
\hline 2 & Dinheiro & 98 & 124 & 222 & 0,00 \\
\hline 3 & Sapato & 25 & 102 & 127 & $30,92^{* * *}$ \\
\hline 4 & Shopping & 48 & 74 & 122 & 1,16 \\
\hline 5 & Gastar & 38 & 36 & 74 & 1,54 \\
\hline 6 & Loja & 33 & 40 & 73 & 0,03 \\
\hline 7 & Comida & 25 & 34 & 59 & 0,08 \\
\hline 8 & Bolsa & 3 & 48 & 51 & $30,35^{* * *}$ \\
\hline 9 & Carro & 32 & 18 & 50 & $7,96^{* *}$ \\
\hline 10 & Diversão & 15 & 18 & 33 & 0,02 \\
\hline 11 & Acessórios & 5 & 28 & 33 & $11,28^{* * *}$ \\
\hline 12 & Prazer & 9 & 21 & 30 & 2,45 \\
\hline 13 & Casa & 14 & 16 & 30 & 0,07 \\
\hline 14 & Livro & 10 & 18 & 28 & 0,82 \\
\hline 15 & Telemóvel & 13 & 15 & 28 & 0,06 \\
\hline 16 & Computador & 19 & 8 & 27 & $7,50 * *$ \\
\hline 17 & Prenda & 9 & 18 & 27 & 1,29 \\
\hline 18 & Marca & 15 & 11 & 26 & 1,92 \\
\hline 19 & Sapatilha & 22 & 4 & 26 & $17,23^{* * *}$ \\
\hline 20 & Necessidade & 12 & 14 & 26 & 0,04 \\
\hline 21 & Dívida & 15 & 11 & 26 & 1,92 \\
\hline 22 & Despesa & 12 & 13 & 25 & 0,15 \\
\hline 23 & Consumismo & 12 & 11 & 23 & 0,59 \\
\hline 24 & Felicidade & 8 & 14 & 22 & 0,55 \\
\hline 25 & Electrónicos & 8 & 14 & 22 & 0,55 \\
\hline 26 & Videojogos & 20 & 1 & 21 & $22,19^{* * *}$ \\
\hline 27 & Alegria & 6 & 15 & 21 & 2,08 \\
\hline 28 & Maquilhagem & 2 & 18 & 20 & $9,48 * *$ \\
\hline 29 & Preço & 10 & 7 & 17 & 1,48 \\
\hline 30 & Filme & 11 & 5 & 16 & $3,91^{*}$ \\
\hline 31 & Satisfação & 6 & 10 & 16 & 0,29 \\
\hline 32 & Jóias & 2 & 13 & 15 & $5,79^{*}$ \\
\hline 33 & Cartão de crédito & 6 & 9 & 15 & 0,11 \\
\hline 34 & Perfume & 2 & 12 & 14 & $5,08^{*}$ \\
\hline
\end{tabular}

${ }^{*} p \leq 0,05 ;{ }^{* *} p \leq 0,01 ; * * * p \leq 0,001$.

Podemos observar que os alunos da escola privada citaram mais as palavras: dinheiro $(p<$
$0,05)$, shopping ( $p<0,001)$, gastar $(p<0,05)$, loja $(p<0,001)$, bolsa $(p<0,01)$, marca $(p<$ 
$0,001)$, despesa $(p<0,05)$, e consumismo ( $p<$ $0,05)$. Por sua vez, os alunos da escola pública citaram mais as palavras: sapato $(p<0,01)$, aces- sórios $(p<0,001)$, casa $(p<0,01)$, telemóvel ( $p$ $<0,01)$, dívida $(p<0,001)$, preço $(p<0,05)$, jóias $(p<0,01)$, e perfume $(p<0,05)$.

Tabela 2

Frequência das Palavras mais Evocadas pelos Respondentes Brasileiros em Função do Tipo de Escola

\begin{tabular}{|c|c|c|c|c|c|}
\hline & Palavras & $\begin{array}{l}\text { Privada } \\
n=272\end{array}$ & $\begin{array}{l}\text { Pública } \\
n=210\end{array}$ & $\begin{array}{c}\text { Total } \\
n=482\end{array}$ & $\begin{array}{c}\chi^{2} \\
(g l=1)\end{array}$ \\
\hline 1 & Roupa & 148 & 135 & 283 & 1,94 \\
\hline 2 & Dinheiro & 141 & 81 & 222 & $4,57^{*}$ \\
\hline 3 & Sapato & 54 & 73 & 127 & $9,95 * *$ \\
\hline 4 & Shopping & 100 & 22 & 122 & $32,43 * * *$ \\
\hline 5 & Gastar & 51 & 23 & 74 & $4,72 *$ \\
\hline 6 & Loja & 61 & 12 & 73 & $21,90 * * *$ \\
\hline 7 & Comida & 28 & 31 & 59 & 1,92 \\
\hline 8 & Bolsa & 39 & 12 & 51 & $8,35^{* *}$ \\
\hline 9 & Carro & 22 & 28 & 50 & 3,13 \\
\hline 10 & Diversão & 17 & 16 & 33 & 0,32 \\
\hline 11 & Acessórios & 8 & 25 & 33 & $13,88 * * *$ \\
\hline 12 & Prazer & 15 & 15 & 30 & 0,50 \\
\hline 13 & Casa & 9 & 21 & 30 & $8,50 * *$ \\
\hline 14 & Livro & 18 & 10 & 28 & 0,71 \\
\hline 15 & Telemóvel & 6 & 22 & 28 & $13,93 * *$ \\
\hline 16 & Computador & 13 & 14 & 27 & 0,75 \\
\hline 17 & Prenda & 11 & 16 & 27 & 3,34 \\
\hline 18 & Marca & 25 & 1 & 26 & $16,71 * * *$ \\
\hline 19 & Sapatilha & 16 & 10 & 26 & 0,28 \\
\hline 20 & Necessidade & 13 & 13 & 26 & 0,43 \\
\hline 21 & Dívida & 5 & 21 & 26 & $14,61 * * *$ \\
\hline 22 & Despesa & 19 & 6 & 25 & $3,91 *$ \\
\hline 23 & Consumismo & 18 & 5 & 23 & $4,47^{*}$ \\
\hline 24 & Felicidade & 13 & 9 & 22 & 0,06 \\
\hline 25 & Electrónicos & 12 & 10 & 22 & 0,03 \\
\hline 26 & Videojogos & 13 & 8 & 21 & 0,26 \\
\hline 27 & Alegria & 10 & 11 & 21 & 0,66 \\
\hline 28 & Maquilhagem & 7 & 13 & 20 & 3,72 \\
\hline 29 & Preço & 5 & 12 & 17 & $5,04^{*}$ \\
\hline 30 & Filme & 7 & 9 & 16 & 1,04 \\
\hline 31 & Satisfação & 8 & 8 & 16 & 0,27 \\
\hline 32 & Jóias & 3 & 12 & 15 & $8,08 * *$ \\
\hline 33 & Cartão de crédito & 11 & 4 & 15 & 1,75 \\
\hline 34 & Perfume & 4 & 10 & 14 & $4,41 *$ \\
\hline
\end{tabular}

$* p \leq 0,05 ; * * \leq 0,01 ; * * * p \leq 0,001$ 


\section{Positividade das Palavras}

Com relação à positividade das palavras evocadas em função do tipo de escola e do sexo de pertença, foi observado apenas um efeito principal da escola de pertença (escola privada: 3,64; escola pública: 3,80$), F(1,447)=6,89$, $p=0,009 ; \eta^{2}=0,01$, indicando que os alunos da escola pública atribuem uma conotação mais positiva às palavras evocadas do que os alunos de escola privada.

\section{Discussão}

De uma forma geral, as palavras mais evocadas pelos adolescentes brasileiros dizem respeito ao meio utilizado para a realização da compra (dinheiro), aos produtos (roupa, sapato, comida, bolsa), e ao ambiente onde a compra é efectuada (shopping e loja).

As palavras significativamente mais citadas, evocadas por mais de $20 \%$ dos adolescentes, expressam bem a representação do consumo do público adolescente. Primeiramente surge a palavra "roupa". O vestuário é a categoria de produto mais adquirida nesta faixa etária (Yaoyuneyong \& George, 2010). A importância do vestuário para este público é evidente, porque o vestuário é um componente essencial de identificação e de expressão da identidade, e é uma condição fundamental para pertencer a um grupo (Santos \& Fernandes, 2006).

A literatura indica que o consumo de roupas nesta faixa etária é motivada pela procura de conformidade com o grupo de amigos a que os adolescentes pertencem ou a que desejam pertencer (Meyer \& Anderson, 2000), pois as roupas servem tanto como instrumento de sedução e conquista do outro, como também surgem como um instrumento de diferenciação social (Gonzaga, 2010; Richins \& Rudmin, 1994). De facto, o que os amigos pensam sobre o adolescente é resultado, principalmente, de uma avaliação das roupas que ele utiliza (Santos \& Fernandes, 2006).

Em segundo lugar, temos a palavra "dinheiro", como o meio utilizado para que a realização da compra seja possível: se não há dinheiro, a compra não pode ser efectuada. $\mathrm{O}$ dinheiro tem uma natureza inerentemente ambivalente, pois ao mesmo tempo que pode ser considerado como um valor, um fim a ser alcançado, ou seja, como um princípio orientador utilizado pelos indivíduos como critério para justificar as suas acções e avaliar pessoas, o dinheiro também pode ser considerado como uma ferramenta, ou seja como um meio para alcançar outros tipos de valores, como o amor, a segurança, e o reconhecimento social (Rinaldi \& Bonanomi, 2011).

Na sociedade capitalista contemporânea, o dinheiro é a grande fonte de poder e estatuto social (Simmel, 2009), que pode ser usado para dar acesso a uma série de produtos e serviços que podem trazer grandes transformações para a vida da pessoa (Richins, 2011). De facto, o dinheiro é o caminho que permite ao indivíduo obter posses (Richins \& Rudmin, 1994).

Em terceiro lugar, temos a palavra "sapato", como uma palavra tipicamente relacionada ao público feminino. As marcas de vestuário e calçados são as marcas com as quais os jovens mais se identificam, e atribuem maior importância, reflectindo a importância que a aparência e a moda têm para esta faixa etária (Galhardo, 2006).

E por fim, temos a palavra "shopping", representando o espaço onde estas compras são realizadas. De facto, os centros comerciais surgiram devido às novas necessidades de consumo decorrentes da modernidade, consagrando-se como verdadeiros santuários da mercadoria (Ferreira, Fonseca, Geraldo, \& Maruno, 2006). Além disso, é um local frequentemente visitado pelo público juvenil, onde os adolescentes realizam as suas compras e procuram sociabilidade (Wypych, 2011).

Relativamente às diferenças encontradas nas palavras evocadas pelos rapazes e raparigas, no que diz respeito ao acto de comprar, verificamos que as palavras estão relacionadas, tipicamente, com o estereótipo social do masculino e do feminino. Os rapazes evocaram produtos com um valor mais elevado, como carro, computador, videojogos, e filme, e as raparigas evocaram produtos relacionados ao uso pessoal com fins de beleza e estética, como roupa, sapato, bolsa, acessórios, maquilhagem e jóias. 
Estes resultados confirmam outros estudos relatados na literatura. Segundo estes estudos, os produtos mais procurados pelos rapazes são sapatilhas, computadores, telemóveis e videojogos (Akcay, 2012; Souza \& Silva, 2006), o que mostra que os homens geralmente se envolvem em compras de produtos relacionados com a tecnologia (electrónicos, CD, DVDs, hardware, software), e com produtos que promovam a sua autonomia (carros, motas) (Dittmar, 1989). Por sua vez, as mulheres compram mais produtos relacionados com a aparência (acessórios, sapatos e roupas; Britsman \& Sjölander, 2011; Coley \& Burgess, 2003).

Neste sentido, um estudo realizado por Beyda (2010) indica também que os rapazes mostram mais interesse em electrónica e estão mais familiarizados com os preços destas categorias de produtos, enquanto as raparigas mostram mais familiaridade com as questões de preço e marca quando se trata de produtos relacionados ao vestuário. Geralmente, pode-se observar que os homens compram produtos relacionados com a identidade pessoal, e as mulheres, relacionados com a identidade social (Coley \& Burgess, 2003), e que os homens compram mais produtos funcionais, e as mulheres, mais produtos estéticos (Dittmar, Beattie, \& Friese, 1996; Rook \& Hoch, 1985).

As diferenças encontradas na frequência das palavras dos alunos de escola pública e da escola privada reflectem também os padrões de consumo da classe social em que os adolescentes estão inseridos. Por um lado, verificamos uma maior frequência das palavras dinheiro, shopping, gastar, loja, marca e consumismo nos alunos da escola privada, que frequentam mais os centros comerciais e têm um rendimento familiar superior. Por outro lado, emergem as palavras dívida, casa, telemóvel e preço, para os alunos da escola pública.

É importante ressaltar que adolescentes com e sem poder aquisitivo têm desejos de consumo diferentes, mas que também há semelhanças, mesmo pertencentes a classes sociais tão distintas (Souza \& Silva, 2006). Por exemplo, observamos que palavras como roupa, carro e computador não apresentaram diferenças signi- ficativas entre os adolescentes de ambas escolas.

Por fim, verificamos que os alunos de escola pública atribuem uma conotação mais positiva às palavras. Pessoas que vivenciam uma maior privação económica tendem a atribuir um maior valor aos bens materiais (Rinaldi \& Bonanomi, 2011). Assim, a actividade de compra é ao mesmo tempo pouco frequente e muito desejada, levando a atribuir uma maior positividade aos conteúdos relacionados com o acto de comprar.

\section{Estudo 2: Portugal}

O estudo realizado em Portugal incidiu sobre o efeito da percepção da crise económica. Começámos por aplicar uma análise factorial em componentes principais sobre os quatro itens propostos para avaliar esta percepção (ver Tabela 3). Depois de retirarmos o item sobre Portugal, que apresentava uma saturação fraca, obtivemos um único factor com valor próprio superior a um, que explica $65,58 \%$ da variância.

\section{Tabela 3}

Solução Factorial da Escala Percepção da Crise

\begin{tabular}{lrc}
\hline $\begin{array}{l}\text { O quanto sentes que a actual } \\
\text { crise económica afecta di- } \\
\text { rectamente... }\end{array}$ & $\begin{array}{c}\text { Média } \\
(D P)\end{array}$ & Saturação \\
\hline $\begin{array}{l}\text { 1. A tua vida } \\
\text { 2. A vida da tua família }\end{array}$ & $4,85(1,84)$ & 0,83 \\
3. A vida de teus amigos & $4,37(1,72)$ & 0,69 \\
4. Portugal & $6,35(1,26)$ & 0,22 \\
\hline
\end{tabular}

Dado o valor do alfa de Cronbach ser suficiente $(\alpha=0,73)$, construímos uma escala de percepção da crise económica e, para constituir dois grupos com percepções diferentes da crise, dividiu-se os respondentes utilizando a mediana $(4,55)$ da escala. Assim, o grupo de menor percepção da crise inclui os respondentes cujas respostas vão de 1 a 4,55 $(n=118)$, e o grupo de maior percepção da crise reúne os cujas respostas vão de 4,56 a $7(n=120)$. Os dois grupos diferenciam-se em todos os itens, como se pode ver na Tabela 4. 
Tabela 4

Diferenças Entre os Grupos com Maior e Menor Percepção da Crise Segundo o Teste t de Student

\begin{tabular}{lccccc}
\hline \multirow{2}{*}{ Dimensões de análise } & \multicolumn{2}{c}{ Percepção da crise } & & \multirow{2}{*}{$t(236)$} \\
\cline { 2 - 3 } & Menor & Maior & & $p$ \\
\hline 1. A tua vida & 2,72 & 5,48 & & 17,48 & $<0,001$ \\
2. A vida da tua família & 3,56 & 6,12 & 14,95 & $<0,001$ \\
3. A vida dos teus amigos & 3,44 & 5,28 & & 9,81 & $<0,001$ \\
\hline
\end{tabular}

Apresentamos de seguida as palavras mais evocadas pelos adolescentes portugueses, e comparamos as frequências das palavras em função do sexo de pertença (masculino vs. feminino), e dos níveis de percepção da crise (menor vs. maior).

\section{Resultado Global}

Recolheu-se, no conjunto, 1122 respostas à questão de saber o que os adolescentes pensam quando pensam em "comprar", entre as quais 120 foram palavras diferentes. $\mathrm{O}$ índice de riqueza é muito baixo $(0,11)$, o que revela de novo uma representação estereotipada do conceito de "comprar". As frequências de evocação variam entre 1 (42 palavras únicas) e 174 ("roupa"). As dez palavras mais citadas pelos adolescentes portugueses como resultado das associações livres foram: roupa $(n=174)$, dinheiro $(n=133)$, comida $(n=95)$, shopping $(n=58)$, sapatilha $(n=$ $43)$, gastar $(n=38)$, jogos $(n=35)$, loja $(n=29)$, sapato $(n=22)$ e desporto $(n=22)$.

\section{Resultado por Sexo}

As cinco palavras mais citadas pelos rapazes portugueses foram: roupa $(n=69)$, dinheiro $(n=63)$, comida $(n=48)$, sapatilha $(n=23) \mathrm{e}$ jogos $(n=23)$. As cinco palavras mais citadas pelas raparigas portuguesas foram: roupa $(n=$ $105)$, dinheiro $(n=70)$, comida $(n=47)$, shopping $(n=39)$ e loja $(n=21$; ver Tabela 5$)$.

A fim de verificar se havia diferenças significativas no que diz respeito à frequência das palavras evocadas pelos alunos do sexo masculino e feminino, aplicou-se um teste de qui-quadrado sobre as palavras que obtiveram frequência igual ou superior a 10 , totalizando 22 palavras.

Das 22 palavras mais evocadas, apenas 8 apresentaram diferenças significativas nas fre- quências relativas ao sexo de pertença. Os rapazes evocaram mais palavras como: desporto $(p<0,001)$, carro $(p<0,001)$ e videojogos $(p<$ $0,001)$, enquanto as raparigas evocaram mais as palavras como: roupa $(p<0,05)$, shopping ( $p<$ $0,05)$, loja $(p<0,05)$, sapato $(p<0,05)$ e acessórios ( $p<0,001$; ver Tabela 5$)$.

\section{Resultado por Níveis de Percepção da Crise}

As cinco palavras mais citadas pelos adolescentes portugueses com menor percepção da crise foram: roupa $(n=84)$, dinheiro $(n=58)$, comida $(n=44)$, shopping $(n=33)$ e sapatilhas $(n=$ 19). As cinco palavras mais citadas pelos adolescentes com maior percepção da crise foram: roupa $(n=90)$, dinheiro $(n=75)$, comida $(n=51)$, shopping $(n=25)$ e gastar $(n=25$; ver Tabela 6$)$.

Com objectivo de verificar se havia diferenças significativas no que diz respeito à frequência das palavras evocadas pelos adolescentes com menor e maior percepção da crise económica, aplicou-se outra vez o teste de qui-quadrado.

Das 22 palavras mais evocadas, encontrou-se apenas uma diferença significativa nas frequências das palavras em função do nível de percepção da crise. Os adolescentes com maior percepção da crise citaram mais a palavra "crise" $(p<0,05$; ver Tabela 6$)$.

\section{Positividade das Palavras}

Relativamente à positividade das palavras evocadas, não foi encontrado um efeito principal do sexo de pertença, $F(1,205)=0,06, n s$, nem um efeito principal da percepção da crise económica, $F(1,205)=0,06, n s$. Tanto os rapazes $(M$ $=3,80 ; D P=0,58)$ como as raparigas $(M=3,79$; 
Tabela 5

Frequência das Palavras mais Evocadas (f $>$ 10) pelos Respondentes Portugueses em Função do Sexo de Pertença

\begin{tabular}{|c|c|c|c|c|c|}
\hline & Palavras & $\begin{array}{c}\text { Rapazes } \\
n=117\end{array}$ & $\begin{array}{c}\text { Raparigas } \\
n=121\end{array}$ & $\begin{array}{c}\text { Total } \\
n=238\end{array}$ & $\begin{array}{c}\chi^{2} \\
(g l=1)\end{array}$ \\
\hline 1 & Roupa & 69 & 105 & 174 & $6,34 *$ \\
\hline 2 & Dinheiro & 63 & 70 & 133 & 0,18 \\
\hline 3 & Comida & 48 & 47 & 95 & 0,07 \\
\hline 4 & Shopping & 19 & 39 & 58 & $6,27^{*}$ \\
\hline 5 & Sapatilhas & 23 & 20 & 43 & 0,32 \\
\hline 6 & Gastar & 19 & 19 & 38 & 0,01 \\
\hline 7 & Jogos & 23 & 12 & 35 & 3,82 \\
\hline 8 & Loja & 8 & 21 & 29 & $5,42 *$ \\
\hline 9 & Sapato & 2 & 20 & 22 & $14,16^{* *}$ \\
\hline 10 & Desporto & 20 & 2 & 22 & $15,31 * *$ \\
\hline 11 & Carro & 19 & 2 & 21 & $14,31 * *$ \\
\hline 12 & Alegria & 13 & 6 & 19 & 2,81 \\
\hline 13 & Telemóvel & 5 & 13 & 18 & 3,31 \\
\hline 14 & Diversão & 11 & 7 & 18 & 1,02 \\
\hline 15 & Casa & 11 & 5 & 16 & 2,45 \\
\hline 16 & Crise & 6 & 8 & 14 & 0,23 \\
\hline 17 & Acessórios & - & 14 & 14 & $15,56^{* *}$ \\
\hline 18 & Videojogos & 14 & - & 14 & $14,46^{* *}$ \\
\hline 19 & Equipa desportiva & 9 & 4 & 13 & 2,09 \\
\hline 20 & Preço & 9 & 4 & 13 & 2,09 \\
\hline 21 & Compras & 8 & 4 & 12 & 1,46 \\
\hline 22 & Poupar & 6 & 5 & 11 & 0,13 \\
\hline
\end{tabular}

$* p \leq 0,05 ; * * p \leq 0,001$

$D P=0,67)$ atribuem uma elevada positividade às conotações das palavras evocadas.

Podemos observar a interacção entre o sexo de pertença e a percepção da crise económica na positividade das palavras, $F(1,205)=4,71, p$ $=0,031 ; \eta^{2}=0,02$. Contudo, uma comparação entre pares de médias não realça diferenças significativas entre os níveis de percepção da crise [menor percepção: rapazes: 3,70; raparigas: $3,89, t(98)=1,82, n s$; maior percepção: rapazes: $3,91$; raparigas: $3,72, t(107)=1,30, n s]$; nem entre os sexos [rapazes: menor percepção: 3,70 ; maior percepção: $3,91, t(100)=1,45, n s$; rapa- rigas: menor percepção: 3,89; maior percepção: $3,72, t(105)=1,64, n s]$.

\section{Discussão}

As palavras mais citadas pelos adolescentes portugueses, semelhantemente ao Estudo 1, referem-se, principalmente, ao meio de efectuar a transacção de compra (dinheiro), aos produtos (roupa, comida, sapatilhas, jogos), e ao ambiente de compra (shopping e loja).

As diferenças encontradas entre os sexos confirmam os resultados encontrados no Estudo 1. 
Tabela 6

Frequência das Palavras mais Evocadas pelos Respondentes Portugueses em Função do Nível de Percepção da Crise

\begin{tabular}{|c|c|c|c|c|c|}
\hline & Palavras & $\begin{array}{c}\text { Menor Percepção } \\
\quad n=118\end{array}$ & $\begin{array}{l}\text { Maior Percepção } \\
\quad n=120\end{array}$ & $\begin{array}{c}\text { Total } \\
n=238\end{array}$ & $\begin{array}{c}\chi^{2} \\
(g l=1)\end{array}$ \\
\hline 1 & Roupa & 84 & 90 & 174 & 0,12 \\
\hline 2 & Dinheiro & 58 & 75 & 133 & 1,90 \\
\hline 3 & Comida & 44 & 51 & 95 & 0,40 \\
\hline 4 & Shopping & 33 & 25 & 58 & 1,24 \\
\hline 5 & Sapatilhas & 19 & 24 & 43 & 0,50 \\
\hline 6 & Gastar & 13 & 25 & 38 & 3,59 \\
\hline 7 & Jogos & 15 & 20 & 35 & 0,63 \\
\hline 8 & Loja & 14 & 15 & 29 & 0,02 \\
\hline 9 & Sapato & 12 & 10 & 22 & 0,22 \\
\hline 10 & Desporto & 15 & 7 & 22 & 3,05 \\
\hline 11 & Carro & 13 & 8 & 21 & 1,28 \\
\hline 12 & Alegria & 9 & 10 & 19 & 0,04 \\
\hline 13 & Telemóvel & 11 & 7 & 18 & 0,96 \\
\hline 14 & Diversão & 8 & 10 & 18 & 0,19 \\
\hline 15 & Casa & 7 & 9 & 16 & 0,22 \\
\hline 16 & Crise & 3 & 11 & 14 & $4,44 *$ \\
\hline 17 & Acessórios & 5 & 9 & 14 & 1,08 \\
\hline 18 & Videojogos & 7 & 7 & 14 & 1,19 \\
\hline 19 & Equipa desportiva & 9 & 4 & 13 & 2,01 \\
\hline 20 & Preço & 9 & 4 & 13 & 2,01 \\
\hline 21 & Compras & 6 & 6 & 12 & 0,00 \\
\hline 22 & Poupar & 6 & 5 & 11 & 0,11 \\
\hline
\end{tabular}

${ }^{*} p \leq 0,05$.

Enquanto os rapazes estão mais relacionados com produtos electrónicos (Beyda, 2010) e de prestígio social (Dittmar, 2011), as raparigas estão mais relacionadas com produtos de estética e ao ambiente de compra (Britsman \& Sjölander, 2011).

Relativamente aos rapazes, os nossos dados corroboram também os resultados encontrados por Silva e Poeschl (2001/2002) que verificaram que, em Portugal, a masculinidade está tipicamente relacionada com o desporto e a bebida. Tais diferenças são resultados de uma construção social e histórica das relações entre os sexos
(Poeschl, Silva, \& Clémence, 2004).

No que diz respeito às diferenças encontradas entre os níveis de percepção da crise, apenas a palavra "crise" apresenta diferença significativa. De forma não surpreendente, os adolescentes que têm uma maior percepção da crise evocaram mais esta palavra. Para além disso, os dados sugerem que os níveis de percepção da crise não afectam de forma significativa os significados atribuídos ao acto de comprar dos adolescentes portugueses. Os adolescentes com uma menor percepção e os adolescentes com uma maior per- 
cepção da crise relacionam as mesmas palavras ao acto de comprar.

\section{Considerações Finais}

A presente investigação teve o objectivo de conhecer os significados que os adolescentes atribuem ao acto de comprar e de identificar eventuais factores de variação nestes significados em função do sexo de pertença e do contexto socioeconómico que os adolescentes vivenciam no seu país. Verificaram-se diferenças entre os sexos no Brasil e em Portugal, e, no Brasil, entre os dois tipos de escola.

De uma forma geral, constatou-se que os significados atribuídos ao acto de comprar são bastante semelhantes nos dois países, e que as palavras mais evocadas pelos adolescentes foram praticamente as mesmas (roupa, dinheiro e shopping). Assim, podemos inferir que, para os adolescentes, comprar significa sobretudo: gastar dinheiro em roupas no shopping.

$\mathrm{Na}$ actualidade os adolescentes têm mais poder de compra do que dantes, o que motiva as lojas a oferecer produtos destinados a esta faixa etária. Os adolescentes tendem a comprar sobretudo roupa dado que o vestuário reveste, nesta faixa etária, um significado particular. Com efeito, como o salientam vários autores (Bouchet, Badaoui, \& Lebrun, 2007; Cassidy \& Schijndel, 2011; Szuster, Casotti, \& Sauerbron, 2014), os adolescentes se encontram num período de construção identitária onde procuram se identificar já não com os pais mas sim com grupos que valorizam. O estilo de vestir e as marcas adoptados permitem-lhes fazer parte de um grupo específico, reconhecível pelos outros, o que explica a razão pela qual a roupa e as marcas tornam-se num dos modos de expressão preferido dos adolescentes (Solomon, 2011). Assim, comprar, para os adolescentes seria essencialmente um comportamento associado à expressão da sua identidade, pessoal e social, definida pelo estilo de roupa que veste.

Os adolescentes compram a sua roupa nos centros comerciais, locais que frequentem por dois objectivos: para fazer compras e para socializar, encontrar amigos, namorar, comer, ir ao cinema (Wypych, 2011). Os centros comerciais não desempenham com efeito apenas a função de ser centros de compras, mas também constituem espaços simbólicos de integração social dos jovens. $O$ facto de comprar a sua roupa nos centros comerciais que costumam frequentar aparece assim como uma característica partilhada pelo público jovem quer do Brasil quer de Portugal. Por conseguinte, independentemente dos contextos socioeconómico em que são inseridos, comprar parece significar basicamente para os adolescentes "comprar roupa no shopping".

\section{Referências}

Akcay, O. (2012). Marketing to teenagers: The influence of color, ethnicity and gender [Special issue]. International Journal of Business and Social Science, 3(2), 10-18.

Amon, D., Guareschi, P., Roso, A., \& Veronese, M. (2010). Psicologia Social e Comunicação Publicitária: Uma análise crítica. Ciências Sociais Unisinos, 46(2), 178-186. doi:10.4013/ csu.2010.46.2.07

Bauman, Z. (2007). Vida para consumo. Rio de Janeiro, RJ: Jorge Zahar.

Beyda, T. (2010). Who teaches them to consume: A study of Brazilian youngsters. International Journal of Consumer Studies, 34(3), 298-305. doi:10.1111/j.1470-6431.2009.00857.x

Bouchet, P., Badaoui, K., \& Lebrun, A. (2007). L'identité sociale au coeur des comportements de consommation des adolescents. Le cas des styles vestimentaires. Actes des 6èmes Journées Normandes sur la Consommation: Sociétéet consommation. Retrieved from http://www.argonautes. $\mathrm{fr} /$ sections.php?op=viewarticle $\&$ artid $=479$

Britsman, S., \& Sjölander, K. (2011). Teenage girls aged 13 to 15 and their impulse consumption of clothes (Bachelor thesis, University of Gothenburg). Retrieved from https://gupea.ub.gu.se/bitstream/2077/25697/1/gupea_2077_25697_1.pdf

Bush, A., Martin, C., \& Bush, V. (2004). Sports celebrity influence of the behavorial intentions of generation Y. Journal of Advertising Research, 44, 108-118.

Camargo, B., \& Bertoldo, R. (2006). Comparação da vulnerabilidade de estudantes da escola pública e particular em relação ao HIV. Estudos de Psicologia (Campinas), 23(4), 369-379. 
Cassidy, T., \& Schijndel, H. (2011). Youth identity ownership from a fashion marketing perspective. Journal of Fashion Marketing \& Management, 15(2), 163-177.

Castro, L. (1998). Infância e adolescência na cultura do consumo. Rio de Janeiro, RJ: NAU.

Chandler, T., \& Heinzerling, B. (1999). Children and adolescents in the marketplace: Twenty-five years of academic research. Ann Arbor, MI: The Pierian Press.

Coley, A., \& Burgess, B. (2003). Gender differences in cognitive and affective impulse buying. Journal of Fashion Marketing and Management, 7(3), 282-295. doi:10.1108/13612020310484834

Costa, M., \& Koslinski, M. (2012). Escolha, estratégia e competição por escolas públicas. ProPosições, 23(2), 195-213.

Crabb, P., \& Bielawski, D. (1994). The social representation of material culture and gender in children's books. Sex Roles, 30(1-2), 69-79.

Deconchy, J. (1971). L'orthodoxie religieuse. Paris: Les Editions Ouvrières.

Dittmar, H. (1989). Gender identity-related meanings of personal possessions. British Journal of Social Psychology, 28(2), 159-171. doi:10.1111/j.2044-8309.1989.tb00857.x

Dittmar, H. (2011). Consumer culture, identity and well-being: The search for the 'good life' and the 'body perfect'. New York: Psychology Press.

Dittmar, H., Beattie, J., \& Friese, S. (1996). Objects, decision considerations and self-image in men's and women's impulse purchases. Acta Psychologica, 93(1-3), 187-206. doi:10.1016/00016918(96)00019-4

Ferreira, C., Fonseca, S., Geraldo, E., \& Maruno, B. (2006). O santuário da mercadoria: Shopping center. Trabalho apresentado no XI Simpósio de Ciências da Comunicação na Região Sudeste, Ribeirão Preto, SP, Brasil.

França, M., \& Gonçalves, F. (2010). Provisão pública e privada de Educação Fundamental: Diferenças de qualidade medidas por meio de propensity score. Economia Aplicada, 14, 373-390. doi:10.1590/S1413-80502010000400006

Gade, C. (1998). Psicologia do consumidor e da propaganda. São Paulo, SP: EPU.

Galhardo, A. (2006). "Marcas com que me identifico" - O ponto de vista de um grupo de jovens consumidores In P. Cardoso, S. Gaio, \& J. Seoane (Eds.), Jovens, marcas e estilos de vida (pp.
225-234). Porto, Portugal: Edições Universidade Fernando Pessoa.

Garófalo, G. (1998). Considerações sobre a microeconomia. In D. Pinho \& M. Vasconcelos (Eds.), Manual de Economia (2. ed., pp. 69-80). São Paulo, SP: Saraiva.

Gonzaga, E., (2010). Shopping center, subjetividade e exclusão social. Fragmentos de Cultura, 20(1), 55-68.

Gunter, B. (2001). As crianças como consumidoras: Uma análise psicológica do mercado juvenil. Lisboa, Portugal: Instituto Piaget.

Hands, D. (2010). Economics, Psychology and the History of consumer choice theory. Cambridge Journal of Economics, 34(4), 633-648. doi:10.1093/cje/bep045

Hall, S. (2002). A identidade em questão. Rio de Janeiro, RJ: DP \& A.

Latif, A., Saleem, S., \& Abideen, Z. U. (2011). Influence of role model on Pakistani urban teenager's purchase behavior. European Journal of Economics, Finance \& Administrative Sciences(31), 7-16.

Lillis, M. (2008). Perspectives in consumer behavior: An anthropological approach. Journal of Consumer Marketing, 25(3), 191-191.

Lloyd, B., \& Duveen, G. (1990). A semiotic analysis of the development of social representations of gender. In G. Duveen \& B. Lloyd (Eds.), Social representations and the development of knowledge (pp. 27-46). New York: Cambridge University Press.

Macinnis, D., \& Folkes, V. (2010). The disciplinary status of consumer behavior: A sociology of science perspective on key controversies. Journal of Consumer Research, 36(6), 899-914.

Mangleburg, T., Doney, P., \& Bristol, T. (2004). Shopping with friends and teens' susceptibility to peer influence. Journal of Retailing, 80(2), 101-116. doi:10.1016/j.jretai.2004.04.005

Meyer, D., \& Anderson, H. (2000). Preadolescents and apparel purchasing: Conformity to parents and peers in the consumer socialization process. Journal of Social Behavior \& Personality, 15(2), 243-257.

Morton, L. (2002). Targeting generation Y. Public Relations Quarterly, 47(2), 46-48.

Moschis, G., Moore, R., \& Stephens, L. (1977). Purchasing patterns of adolescent consumers. Journal of Retailing, 53(1), 17. 
Noble, S., Haytko, D., \& Philips, J. (2009). What drives college-age generation Y consumers? Journal of Business Research, 62(6), 617-628.

Ozella, S., \& Aguiar, W. (2008). Desmistificando a concepção de adolescência. Cadernos de Pesquisa, 38(133), 97-125.

Pasdiora, M., \& Brei, V. (2014). A formação do hábito de consumo infantil: Uma análise crítica da Teoria de Consumo de Status aplicada às classes sociais altas e baixas no Brasil. Organizações \& Sociedade, 21(68), 789-814.

Poeschl, G. (1992). L'Intelligence: un concept à la recherche d'un sens (Thèse de doctorat, Université de Genéve, Suisse).

Poeschl, G., Silva, A., \& Clémence, A. (2004). Representações da maculinidade e da feminilidade e retratos de homens e de mulheres na literatura portuguesa. Psicologia: Revista da Associação Portuguesa Psicologia, 18(1), 31-46.

Richins, M. (2011). Materialism, transformation expectations, and spending: Implications for credit use. Journal of Public Policy \& Marketing, 30(2), 141-156. doi:10.1509/jppm.30.2.141

Richins, M., \& Rudmin, F. (1994). Materialism and economic psychology. Journal of Economic Psychology, 15(2), 217-231. doi:10.1016/01674870(94)90001-9

Rinaldi, E., \& Bonanomi, A. (2011). Adolescents and money values and tools to handle the future. Italian Journal of Sociology of Education, 3(3), 86-121.

Roland-Levy, C., Boumelki, F., \& Guillet, E. (2010). Representation of the financial crisis: Effect on social representations of savings and credit. Journal of Socio-Economics, 39(2), 142-149. doi:10.1016/j.socec.2010.02.014

Rook, D., \& Hoch, S. (1985). Consuming impulse. Advances in Consumer Research, 12, 23-27.

Rosenberg, S., \& Jones, R. (1972). A method for investigating and representing a person's implicit theory of personality: Theodore Dreiser's view of people. Journal of Personality \& Social Psychology, 22(3), 372-386.

Sampaio, B., \& Guimarães, J. (2009). Diferenças de eficiência entre ensino público e privado no Brasil. Economia Aplicada, 13(1), 45-68. doi:10.1590/S1413-80502009000100003

Santos, C., \& Fernandes, D. (2006). Avaliação da escala Consumer Style Inventory (CSI) no contexto brasileiro. RAM - Revista de Administração Mackenzie, 7(2), 11-38.
Santos, C., \& Fernandes, D. (2011). A socialização de consumo e a formação do materialismo entre os adolescentes. RAM - Revista de Administracao Mackenzie, 12(1), 169-203.

Shannahan, K., Shannahan, R., Bush, A., \& Rocco, R. (2011). Gender as a moderator of role model influence and adolescents' consumer-related behavioral intentions. Marketing Management Journal, 21(1), 47-59.

Shim, S., Serido, J., \& Barber, B. (2011). A consumer way of thinking: Linking consumer socialization and consumption motivation perspectives to adolescent development. Journal of Research on Adolescence, 21(1), 290-299. doi:10.1111/ j.1532-7795.2010.00730.x

Silva, A., \& Poeschl, G. (2001/2002). Representações das semelhanças e das diferenças entre os sexos. Cadernos de Consulta Psicológica, 1718, 153-159.

Simmel, G. (2009). Psicologia do dinheiro e outros ensaios. Lisboa, Portugal: Texto \& Grafia.

Solomon, M. (2011). O comportamento do consumidor: Comprando, possuindo e sendo. Porto Alegre, RS: Bookman.

Souza, A., \& Silva, C. (2006). O consumo na vida de adolescentes de diferentes condições socioeconômicas: Uma reflexão para o marketing no Brasil. Cadernos EBAPE, 4(1), 1-18.

Szuster, F., Casotti, L., \& Sauerbron, J. (2014). Lá vai o consumidor pela ponta esquerda! Um estudo sobre consumo adolescente de futebol na cidade do Rio de Janeiro. Revista do Mestrado em Administração e Desenvolvimento Empresarial da Universidade Estácio de Sá, 8(1), 79-104.

Wang, I., Holloway, B., Beatty, S., \& Hill, W. (2007). Adolescent influence in family purchase decisions: An update and cross-national extension. Journal of Business Research, 60(11), 1117-1124.

Ward, S. (1974). Consumer socialization. Journal of Consumer Research, 1, 1-14.

Wypych, P. (2011). Shopping center: Um espaço de lazer e socialização para o adolescente. Mediação, 13(13), 137-151.

Yaoyuneyong, G., \& George, B. (2010). Impulse buying of apparel products: A study conducted among midwestern teenagers in the United States. Journal of Marketing Trends, 2(1), 5-20.

Recebido: 14/02/2014

$1^{a}$ revisão: $13 / 08 / 2014$

$2^{a}$ revisão: $14 / 08 / 2014$ Aceite final: 16/08/2014 